\title{
La evaluación de la competencia "Comunicación efectiva” a través de rúbricas para estrategias activas: el debate y la presentación de trabajos académicos.
}

\author{
P. Amparo López-Jiménez ${ }^{a}$ Modesto Pérez-Sánchez ${ }^{a}$, \\ a Departamento de Ingeniería Hidráulica y Medio Ambiente. Universitat Politècnica de València. \\ palopez@upv.es; mopesan1@upv.es.
}

\begin{abstract}
The efective communication transmits a clear and accurate message, that allows receptor to assimilate the information When a student is training, he/she has to transmite his/her ideas, projects or methods to rest of public. The transversal competence "Effective communication" is crucial in all phases, althought this competence is most important in last degrees or master courses, when the students are doing their final thesis of Bachelor or Master. The current research describes a learning strategy that was developed in one master subject, called "Polution dispersion in water and atmosphere". This subject is dicted in the second course of the Industrial Engineering Master Degree in ETSII (UPV). The research shows the results in two different periodes, when the acquisition results were excellent.
\end{abstract}

Keywords: effective comunication; contaminant dispersion; public exposition; rubbric

\section{Resumen}

La comunicación efectiva es aquella que transmite un mensaje claro y preciso y que permite al receptor asimilar dicho mensaje. Dentro de la formación de un estudiante, cualquiera que sea su formación, es necesario que sus ideas, proyectos o metodologías sepa transmitirlas al público potencial que se vea expuesto. Aunque es crucial en todas las fases, la competencia transversal "Comunicación efectiva" cobra una importancia esencial en los últimos cursos de grado o máster para la preparación en la defensa de los trabajos final de grado o máster. La presente comunicación expone la estrategia de aprendizaje llevada a cabo en la asignatura "Dispersión de Contaminantes en Agua y Atmósfera" en segundo curso del Máster en Ingeniería Industrial de la ETSII (UPV) y los resultados obtenidos en los dos cursos en los que se ha llevado a cabo, con un éxito tanto en el grado de adquisición de los resultados de aprendizaje como satisfacción del alumnado.

Palabras clave: Comunicación efectiva; Dispersión de contaminantes; exposición pública; rúbrica 


\section{Introducción}

La competencia Comunicación Efectiva, tal como se define en el proyecto de competencias transversales de la UPV (UPV, 2018) "Es la capacidad para transmitir conocimientos y expresar ideas y argumentos de manera clara, rigurosa y convincente, tanto de forma oral como escrita, utilizando los recursos gráficos y los medios necesarios adecuadamente, y adaptándose a las características de la situación y de la audiencia."

Una comunicación efectiva, por tanto, pasa por transmitir conceptos claros de una forma concisa y específica, de manera que los interlocutores capten la mayor parte del mensaje. En este sentido, la consideración de la comunicación efectiva como una competencia transversal en fases finales de la educación superior de los alumnos es una herramienta potente. Esta desarrolla en los alumnos una habilidad importante para su actividad profesional, ya cercana para ellos en los últimos cursos de máster.

Así pues, la comunicación efectiva es una vía para que los alumnos practiquen su capacidad de ser eficaces en el proceso de transmisión de información, muy específica cuando ya estos estudiantes se encuentran en sus últimas etapas. ¿Y qué conocimientos pueden comunicarse? ¿Pueden comunicarse también argumentos e ideas estructuradas para conseguir una opinión del resto de compañeros? Estas preguntas son las que se hacen los docentes que deciden proponer estrategias activas, en las que el alumno se involucre en la preparación de los contenidos a comunicar con el resto de sus compañeros. Más allá de las preguntas de cuáles son las posibles estrategias activas en las que los alumnos puedan involucrarse para la preparación de los contenidos, se encuentra la pregunta de cómo evaluar finalmente la competencia relacionada con la efectividad de la comunicación, mientras los alumnos desarrollan estas estrategias. Los autores de la presente contribución proponemos la utilización de rúbricas para tener en cuenta los diferentes aspectos que en ciertas actividades pueden involucrar una correcta comunicación efectiva, mezclada con la utilización de diferentes metodologías activas que en este caso han sido el debate y el desarrollo de trabajos finales en el desarrollo de la asignatura "Dispersión de Contaminantes en Agua y Atmósfera” en segundo curso del Máster en Ingeniería Industrial, desarrollado en la Escuela Técnica Superior de Ingenieros Industrial de la Universitat Politècnica de València. El propio análisis de cómo puede el problema evaluarse a través de ítems concretos, es en sí, una actividad novedosa para el profesorado. A juicio de los autores, la estrategia pone en valor la capacidad de las rúbricas para evaluar todo tipo de competencias.

\section{Objetivos}

El objetivo de la presente contribución es mostrar las rúbricas desarrolladas para la evaluación de la competencia transversal "Comunicación efectiva” implementadas en la asignatura "Dispersión de Contaminantes en Agua y Atmósfera" en segundo curso del Máster en Ingeniería Industrial de la ETSII (UPV) y los resultados obtenidos en los dos cursos en los que se ha llevado a cabo. La mejora en las capacidades de comunicación efectiva a través de estrategias diversas prepara a los alumnos hacia la presentación de su

(cc) BY-NC-ND 2018, Universitat Politècnica de València

Congreso IN-RED (2018) 
trabajo final de grado o máster mejorando su comunicación oral o escrita, en fases ya cercanas a esta fase de finalización de sus titulaciones.

El desarrollo de estas rúbricas se encuentra dentro del Proyecto de Innovación y Mejora Docente "Desarrollo de Metodologías de Evaluación y Diseño de Rúbricas de la CT-05 Diseño y Proyecto" aplicado al campo de la Ingeniería Hidráulica (Fase II), el cual se encuentra integrado el EICE Desarrollo de Metodologías Activas de evaluación aplicadas al campo de la Ingeniería Hidráulica (DESMAHIA).

\section{Desarrollo de la innovación docente}

El debate y la preparación de trabajos especializados como estrategia activa para el aprendizaje de las asignaturas de últimos cursos de máster.

\section{El debate}

El debate es una estrategia que permite a los alumnos manifestar y presentar sus conocimientos profundos de un tema, a la vez que estimula su espíritu crítico. Permite que los alumnos practiquen la argumentación, fundamentada en una documentación previa por su parte en fuentes fidedignas. Esto debe ser fundamentado en una investigación de los alumnos para fundamentar la tesis que tienen que defender en el mismo. De otro lado, los alumnos tienen que mejorar su capacidad de expresión oral, de improvisación y de ingenio al adaptarse rápidamente a las intervenciones de otros (López-Jiménez y Pérez-Sánchez, 2017a).

El debate permite a los alumnos conseguir el conocimiento profundo de los temas a los que se enfrentan, a la vez que desarrollar sus habilidades expositivas y de representación y análisis de problemas. Algunos de los objetivos docentes conseguidos con el debate son los siguientes (Rodriguez, 2010):

- Utilizar el leguaje específico de forma correcta, argumentando sus ideas con un vocabulario adecuado a los conceptos presentados. Asimismo, esto se consigue de forma mucho más eficiente cuando la temática que se desarrolla se presenta en una lengua que no es la materna para los alumnos.

- Presentar los conceptos con una calidad argumental, que permita convencer a sus compañeros del rol que han adoptado para defender sus posturas y describir sus argumentos. Particularmente ligado a aspectos ambientales de contaminación de agua o aire, siempre controvertidos.

- Defender el contenido de los argumentos presentados a través de fuentes bibliográficas con verosimilitud de forma que se validen, dichos argumentos, a través de la confianza científica de fuentes fidedignas y reconocidas por todos los participantes.

- Demostrar el dominio del tema abordado, con lo que se desarrolla por parte de los ponentes de cada uno de los roles un conocimiento específico del tema presentado. Este conocimiento fundado con una toma de contacto en muchas fuentes, todas 
ellas documentadas, propone un aprendizaje motivador y que, en la experiencia de los autores, permanece fuertemente presente en la memoria de los alumnos.

- Ser capaces de cambiar la opinión de un "oponente" o un auditorio sobre un tema en que se ha propuesto un desarrollo argumentativo.

\section{El desarrollo de trabajos académicos como parte de la profundización en nuevos contenidos.}

El trabajo académico en el contexto de las asignaturas a concurso, se desarrolla como una exposición por parte del alumno de un tema trabajado por el mismo, con mayor profundidad que se presenta en clase de manera argumentativa. Este tema se defiende a través de una presentación argumentativa frente al resto de la clase. Constituye una forma muy interesante de evaluación, puesto que permite al alumno, desarrollar varias destrezas. De un lado, hace que el alumno desarrolle un aprendizaje autónomo basado en la documentación de fuentes fidedignas que debe consultar; de otro, debe desarrollar su capacidad de organizar argumentos, comunicarse, esquematizar y organizar su tiempo.

Además, esta estrategia es muy útil en las asignaturas de nivel de máster, y la asignatura de dispersión de contaminantes en agua y atmósfera no es ajena. La experiencia con alumnos de último trimestre demuestra, a juicio de los autores, que su experiencia argumentativa y de comunicación todavía no se encuentra madura. En este sentido, la dedicación puesta a desarrollar y presentar estos trabajos meticulosamente, es muy útil y bien valorada por los alumnos. La evaluación de estos trabajos, incluida la competencia referida a la comunicación, se realiza a través de rúbricas, conocidas por los alumnos, para que la evaluación sea más efectiva y con unos criterios totalmente objetivos.

\section{La propuesta de rúbricas para la evaluación de las competencias transversales.}

Las rúbricas son guías de puntuación usadas en la evaluación del rendimiento de los estudiantes que describen las características específicas de un producto, proyecto o tarea en varios niveles de funcionamiento, con el fin de clarificar lo que se espera del trabajo del alumno, de valorar su ejecución y de facilitar la proporción de retroalimentación, (Fernández March, 2010).

Las rúbricas como guías de evaluación son particularmente útiles tanto para el profesor, al que permiten observar bajo criterios objetivos, la marcha de los alumnos y adaptarlas de una forma versátil a cada aspecto a evaluar; como para los alumnos, ya que permiten de antemano saber cuál es el nivel que se espera de cada tarea para conseguir una evaluación determinada.

En este sentido, las rúbricas se han desarrollado teniendo en cuenta que los alumnos son de tercer nivel, ya en una etapa final de máster y que las diferentes actividades van encaminadas no sólo a la comunicación efectiva, sino a la adquisición de conocimientos y argumentación especializada en el contexto de los contenidos de la asignatura.

(cc) EY-NC-ND 2018, Universitat Politècnica de València

Congreso IN-RED (2018) 


\section{Resultados}

La propuesta de las rúbricas aquí desarrolladas ha sido implementada durante dos cursos con anterioridad. Asimismo, debe contextualizarse en ambas actividades cual ha sido el carácter de los contenidos a evaluar, junto con la competencia referida a la comunicación efectiva.

Cabe indicar que esta competencia, no solo se evalúa en el desarrollo de esta asignatura, sino que también se trabaja. Cuando comienza cada clase, los alumnos de forma voluntaria se han preparado una presentación breve de un tema relacionado con los contenidos de dicho tema y se aplican las técnicas de comunicación audiovisual que, a juicio de la profesora y el resto de alumnos, son más efectivas para transmitir el mensaje. En experiencia de los autores, esta actividad voluntaria, guiada en las primeras iteraciones y más independiente en las últimas, se convierte en una rutina participativa que tiene muy buena acogida entre los alumnos. Al ser grupos poco numerosos ( 6 alumnos en el curso 2015-2016 y 10 alumnos en 2016-2107), cada alumno ha tenido ocasión de realizar varias presentaciones breves en castellano o inglés antes de que se lleve a cabo el debate o la presentación del trabajo académico de la asignatura.

\section{Rubrica para evaluación de la comunicación efectiva implementada en la actividad "Debate".}

Es importante describir cómo se presenta la actividad en el seno de la asignatura en que se desarrolla. Los alumnos son de último curso y segundo cuatrimestre de Master Ingeniero Industrial en la especialidad medioambiental. Se desarrolla un debate alrededor de la pregunta “¿Es el cambio climático una consecuencia directa de la actividad antropogénica?.

Los alumnos deben posicionarse en dos grupos, uno de ellos argumentar a favor y otros en contra. Se realiza una preparación de la argumentación por parte de ambos grupos y, tras un tiempo de preparación con ayuda de la bibliografía y cualesquiera consulta que desee hacerse, tiene lugar el debate. Los grupos son de entre cuatro y seis alumnos. Cada uno de ellos hace una presentación breve de su postura durante cinco minutos. Para ello se han preparado en equipo, de forma que la argumentación se complementa entre todos los integrantes del mismo grupo.

Terminada esta fase por parte de ambas facciones, se produce un turno de preguntas para defender las dudas que hayan quedado sobre los argumentos. Terminado el turno de preguntas, se produce una votación secreta para determinar cuál de los ponentes (de ambos grupos) ha sido más convincente, mejor argumentador y comunicador.

Por parte de los profesores, se ha desarrollado una rúbrica para evaluar la competencia trasversal de comunicación efectiva, descrita en la Tabla 1. En este caso solamente se han considerado los aspectos relacionados con la comunicación oral.

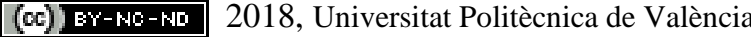


A modo de anécdota, cabe destacar que, en los dos cursos previos, los alumnos han presentado sus opciones y en ambas ocasiones ha resultado más valorada en su capacidad de comunicación y defensa argumental, aquella que defendía que el cambio climático no es consecuencia de la acción antropogénica.

Tabla 1. Rúbrica para la evaluación de la competencia Comunicación efectiva en la asignatura "Dispersión de contaminantes en agua y atmósfera" a través de la actividad: Debate

\begin{tabular}{|c|c|c|c|c|}
\hline \multirow[b]{2}{*}{ INDICADOR } & \multicolumn{4}{|c|}{ DESCRIPTOR } \\
\hline & $\begin{array}{c}\text { D. No } \\
\text { alcanzado }\end{array}$ & C. En desarrollo & B. Adecuado & A. Excelente \\
\hline $\begin{array}{c}\text { 1. Capacidad } \\
\text { de } \\
\text { comunicación } \\
\text { audiovisual }\end{array}$ & $\begin{array}{l}\text { No se ha } \\
\text { preparado una } \\
\text { argumentación } \\
\text { en el rol que ha } \\
\text { adoptado. } \\
\text { No presenta } \\
\text { fundamentos. }\end{array}$ & $\begin{array}{l}\text { No conoce bien el } \\
\text { tema en el rol que } \\
\text { ha adoptado y } \\
\text { cuando lo } \\
\text { presenta no es } \\
\text { convincente. } \\
\text { Presenta pocos } \\
\text { argumentos. }\end{array}$ & $\begin{array}{l}\text { Conoce el tema en } \\
\text { el rol que ha } \\
\text { adoptado, y cuando } \\
\text { lo presenta es } \\
\text { convincente } \\
\text { Presenta } \\
\text { argumentos a favor } \\
\text { de su posura, sin } \\
\text { fundamentar }\end{array}$ & $\begin{array}{l}\text { Demuestra un completo } \\
\text { dominio del tema en el rol } \\
\text { que ha adoptado. No } \\
\text { presenta fisuras en la } \\
\text { argumentación } \\
\text { Presenta muchos } \\
\text { argumentos a favor de su } \\
\text { postura, bien } \\
\text { fundamentados }\end{array}$ \\
\hline $\begin{array}{l}\quad 2 . \\
\text { Habilidades } \\
\text { lingüisticas }\end{array}$ & $\begin{array}{l}\text { No se entiende } \\
\text { bien su } \\
\text { pronunciación, } \\
\text { con tono y } \\
\text { volumen no } \\
\text { adecuados a la } \\
\text { defensa de una } \\
\text { argumentación } \\
\text { en debate }\end{array}$ & $\begin{array}{l}\text { Tiene modos } \\
\text { monótonos de } \\
\text { hablar, con mala } \\
\text { pronunciación, } \\
\text { sin controlar el } \\
\text { énfasis en la } \\
\text { argumentación, } \\
\text { con coletillas }\end{array}$ & $\begin{array}{c}\text { Habla } \\
\text { acpetablemente. } \\
\text { Usa un tono claro y } \\
\text { buena } \\
\text { pronunciación, sin } \\
\text { controlar el énfasis } \\
\text { en la argumentación }\end{array}$ & $\begin{array}{l}\text { Habla perfectamente. En } \\
\text { un tono claro y buena } \\
\text { pronunciación, } \\
\text { enfatizando su } \\
\text { argumentación. }\end{array}$ \\
\hline $\begin{array}{l}\text { 3. Lenguaje } \\
\text { corporal }\end{array}$ & $\begin{array}{l}\text { No tiene buena } \\
\text { postura. No } \\
\text { establece } \\
\text { contacto visual } \\
\text { con el auditorio. } \\
\text { No gestualiza. }\end{array}$ & $\begin{array}{l}\text { Tiene una postura } \\
\text { inexpresiva. No } \\
\text { muestra } \\
\text { entusiasmo por el } \\
\text { rol que defiende. } \\
\text { No tiene } \\
\text { argumentación } \\
\text { gestual. }\end{array}$ & $\begin{array}{c}\text { Tiene buena postura } \\
\text { y establece contacto } \\
\text { visual con el } \\
\text { auditorio la mayoria } \\
\text { del tiempo. } \\
\text { Tiene } \\
\text { argumentación } \\
\text { gestual, con poco } \\
\text { énfasis en las ideas } \\
\text { fuerza. }\end{array}$ & $\begin{array}{c}\text { Tiene buena postura. Se } \\
\text { encuentra relajado y } \\
\text { seguro en el rol que ha } \\
\text { adoptado } \\
\text { Establece contacto visual } \\
\text { con todo el auditorio, } \\
\text { particularmente con su } \\
\text { interlocutor en cada } \\
\text { argumento, apoyando las } \\
\text { ideas fuerza con gestos. }\end{array}$ \\
\hline $\begin{array}{l}\text { 4. Control del } \\
\text { tiempo }\end{array}$ & $\begin{array}{c}\text { No muestra } \\
\text { interés en la } \\
\text { coordinación del } \\
\text { tiempo como } \\
\text { parte de la }\end{array}$ & $\begin{array}{c}\text { Expone las ideas } \\
\text { desordenadas en } \\
\text { tiempos } \\
\text { demasiado cortos } \\
\text { o largos. }\end{array}$ & $\begin{array}{c}\text { Expone las ideas } \\
\text { desordenadas, en } \\
\text { tiempos adecuados. }\end{array}$ & $\begin{array}{c}\text { Expone las ideas } \\
\text { perfectamente ordenadas } \\
\text { en los tiempos previstos } \\
\text { para cada turno de } \\
\text { argumentación y diálogo. }\end{array}$ \\
\hline
\end{tabular}

(c) EY-NC-ND 2018, Universitat Politècnica de València 


\begin{tabular}{|c|c|l|l|}
\hline & presentación de & & \\
ideas ni de & & & \\
preguntas en & & & \\
fase de diálogo & & & \\
argumentativo & & & \\
\hline
\end{tabular}

Rubrica para evaluación de la comunicación efectiva implementada en la actividad “Trabajo académico".

Los trabajos académicos desarrollados por los alumnos en cada curso son propuestos por el profesorado, ya que los contenidos son complementarios a los presentados por los descriptores generales de la asignatura.

Para ilustrar este epígrafe como muestra, algunos de los trabajos académicos presentados por los alumnos en los cursos anteriores han versado sobre las siguientes temáticas:

- Modelos comerciales de dispersión de contaminantes en la atmósfera.

- Modelos comerciales de dispersión de contaminantes en aguas superficiales.

- Modelos comerciales de dispersión de contaminantes en aguas subterráneas.

- Accidentes graves de contaminación de aguas subterráneas: el accidente de Aznalcóllar.

- Accidentes graves de contaminación de aguas marinas: El accidente del Prestige.

- Normativa de análisis de dispersión de contaminantes en atmósfera.

- Normativa relacionada con la calidad de aguas superficiales.

- Emisarios submarinos en España. Principales diseños y casos reales.

- El diseño de la chimenea industrial. Casos reales que presentan las mayores chimeneas del mundo.

- La desalación como sistema de producción de agua potable y su impacto ambiental en relación con la dispersión de salmuera

Los trabajos se presentan durante 15 minutos, y a continuación se da un turno de preguntas. La Tabla 2 describe la rúbrica que los autores de la presente contribución han desarrollado para la evaluación la capacidad efectiva de comunicación, en su parte oral, para la presentación de estos trabajos académicos. Cabe destacar que, secuencialmente, el debate se realiza al final del primer parcial, mientras que la presentación de los trabajos académicos ocurre a 1 final del segundo parcial, de manera que los alumnos han tenido ya más ocasión de trabajar la competencia.

En cuanto a la valoración de la competencia transversal a lo largo de los dos años en que los alumnos han desarrollado los debates y trabajos académicos, los resultados han sido excelentes. Cabe destacar que estos alumnos han trabajado intensamente esta competencia transversal en fases anteriores e incluso con presentaciones breves en la presente asignatura.

(c)) EY-NC-ND 2018, Universitat Politècnica de València 
Esto ha hecho que los resultados hayan sido muy buenos en los dos años en que se han implementado las presentes rúbricas.

Tabla 2. Rúbrica para la evaluación de la competencia Comunicación efectiva en la asignatura "Dispersión de contaminantes en agua y atmósfera” a través de la actividad: Trabajos académicos.

\begin{tabular}{|c|c|c|c|c|}
\hline \multirow[b]{2}{*}{ INDICADOR } & \multicolumn{4}{|c|}{ DESCRIPTOR } \\
\hline & D. No alcanzado & $\begin{array}{c}\text { C. En } \\
\text { desarrollo }\end{array}$ & B. Adecuado & A. Excelente \\
\hline $\begin{array}{l}\text { 1. Capacidad } \\
\text { de } \\
\text { comunicación } \\
\text { audiovisual }\end{array}$ & $\begin{array}{l}\text { No se ha preparado } \\
\text { una presentación } \\
\text { clara. No hay indice, } \\
\text { ni transparencias } \\
\text { coherentes, los } \\
\text { tamaños de letra } \\
\text { impiden entender el } \\
\text { contenido. }\end{array}$ & $\begin{array}{l}\text { No conoce bien } \\
\text { el tema y la } \\
\text { presentación es } \\
\text { mediocre } \\
\text { Se sigue con } \\
\text { dificultad, a } \\
\text { pesar de tener } \\
\text { transparencias. }\end{array}$ & $\begin{array}{l}\text { Conoce el tema que ha } \\
\text { peparado. La } \\
\text { presentación se sigue } \\
\text { facilmente y tiene un } \\
\text { aspecto gráfico y } \\
\text { cientifico adecuado. }\end{array}$ & $\begin{array}{c}\text { Demuestra un completo } \\
\text { dominio del tema que } \\
\text { presenta. La } \\
\text { argumentación es } \\
\text { perfecta en el campo } \\
\text { audiovisual con figuras } \\
\text { y tamaños de letras } \\
\text { legibles y bien } \\
\text { graficadas. }\end{array}$ \\
\hline $\begin{array}{l}2 . \\
\text { Habilidades } \\
\text { lingüisticas }\end{array}$ & $\begin{array}{l}\text { No se entiende bien } \\
\text { su pronunciación, con } \\
\text { tono y volumen no } \\
\text { adecuados a la } \\
\text { defensa de una } \\
\text { argumentación en un } \\
\text { trabajo específico. }\end{array}$ & $\begin{array}{c}\text { Tiene modos } \\
\text { monótonos de } \\
\text { hablar, con mala } \\
\text { pronunciación, } \\
\text { sin controlar el } \\
\text { énfasis en la } \\
\text { presentación de } \\
\text { ideas, con } \\
\text { coletillas }\end{array}$ & $\begin{array}{l}\text { Habla aceptablemente. } \\
\text { Usa un tono claro y } \\
\text { buena pronunciación, } \\
\text { sin controlar el énfasis } \\
\text { en la descripción de } \\
\text { ideas }\end{array}$ & $\begin{array}{l}\text { Habla perfectamente. } \\
\text { En un tono claro y } \\
\text { buena pronunciación, } \\
\text { enfatizando su } \\
\text { argumentación. Motiva } \\
\text { al auditorio sobre el } \\
\text { tema que presenta a } \\
\text { traves de la modulacion } \\
\text { de su lenguaje oral. }\end{array}$ \\
\hline $\begin{array}{l}\text { 3. Lenguaje } \\
\text { corporal }\end{array}$ & $\begin{array}{l}\text { No tiene buena } \\
\text { postura. No establece } \\
\text { contacto visual con el } \\
\text { auditorio. No } \\
\text { gestualiza. }\end{array}$ & $\begin{array}{l}\text { No tiene buena } \\
\text { postura. No } \\
\text { muestra } \\
\text { entusiasmo por } \\
\text { el tema que está } \\
\text { presentando. }\end{array}$ & $\begin{array}{c}\text { Tiene buena postura y } \\
\text { establece contacto } \\
\text { visual con el auditorio } \\
\text { la mayoria del tiempo } \\
\text { Tiene argumentación } \\
\text { gestual, pero no apoya a } \\
\text { las ideas fuerza. }\end{array}$ & $\begin{array}{l}\text { Tiene buena postura. Se } \\
\text { encuentra relajado y } \\
\text { seguro en el conteido y } \\
\text { lenguaje de su } \\
\text { presentación. } \\
\text { Establece contacto } \\
\text { visual con todo el } \\
\text { audotorio, } \\
\text { particularmente con su } \\
\text { interlocutor en cada } \\
\text { argumento, apoyando } \\
\text { las ideas fuerza con } \\
\text { gestos y modos de } \\
\text { comunicarse. }\end{array}$ \\
\hline $\begin{array}{l}\text { 4. Control del } \\
\text { tiempo }\end{array}$ & $\begin{array}{l}\text { No muestra interés en } \\
\text { la coordinación del } \\
\text { tiempo como parte de } \\
\text { la presentación de } \\
\text { ideas }\end{array}$ & $\begin{array}{c}\text { Expone las } \\
\text { ideas en tiempos } \\
\text { demasiado } \\
\text { cortos o largos }\end{array}$ & $\begin{array}{l}\text { Expone las ideas } \\
\text { desordenadas, pero en } \\
\text { tiempos adecuados. }\end{array}$ & $\begin{array}{l}\text { Expone las ideas } \\
\text { perfectamente } \\
\text { ordenadas en los } \\
\text { tiempos previstos para } \\
\text { cada punto de su } \\
\text { presentación } \\
\text { audiovisual, incluidos } \\
\text { antecedentes y } \\
\text { conclusiones. }\end{array}$ \\
\hline
\end{tabular}

(cc) EY-NC-ND 2018, Universitat Politècnica de València 
Las Figuras 1 y 2 muestran los resultados obtenidos por los 16 alumnos de los cursos anteriores en cuanto a la aplicación de las rúbricas para su evaluación en ambas actividades.

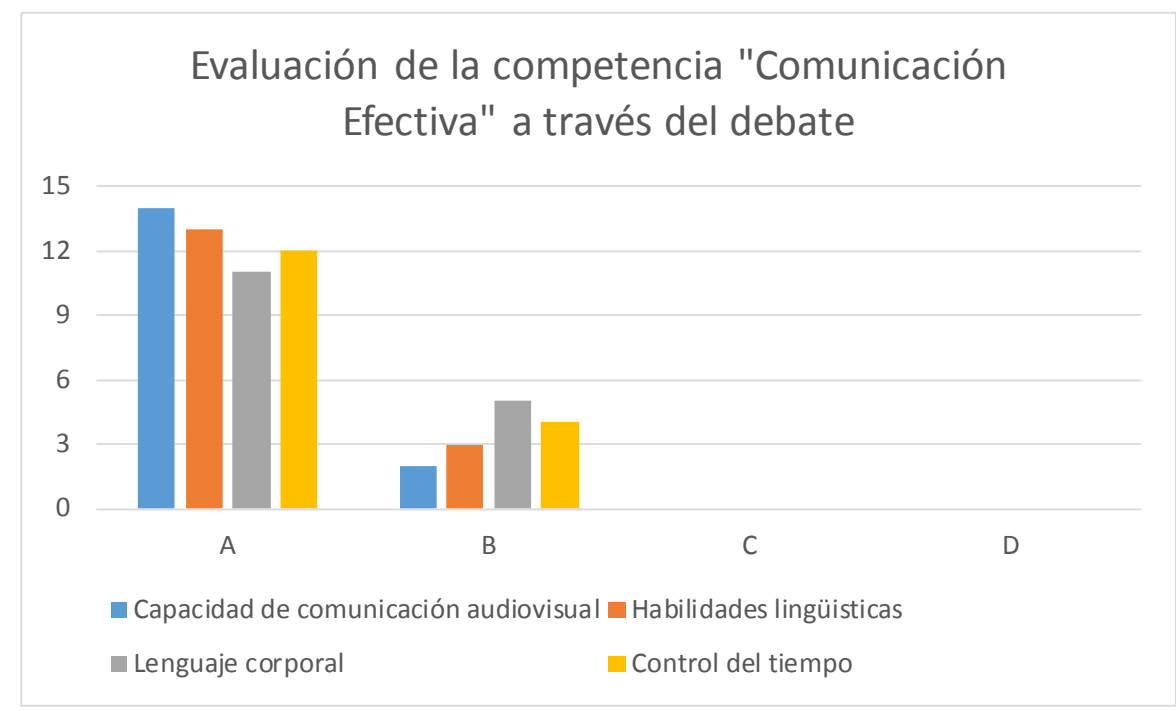

Figura 1. Resultados de la aplicación de la rúbrica de la Tabla 1 en los dos cursos anteriores sobre la actividad Debate.

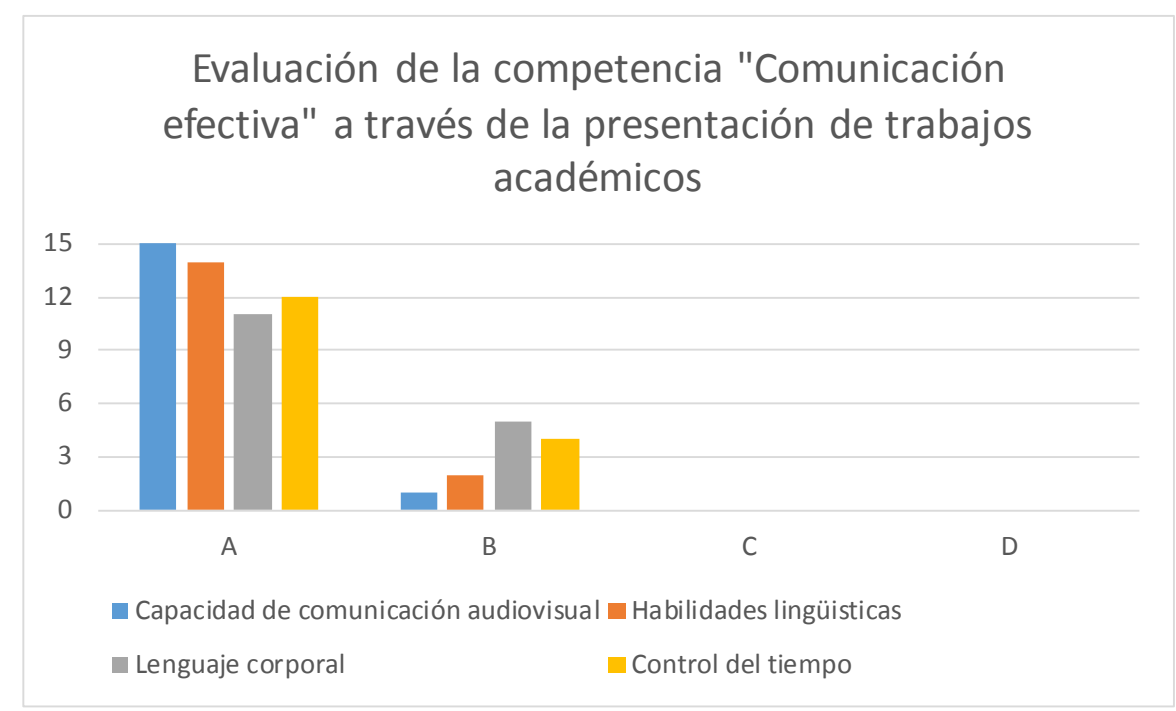

Figura 2. Resultados de la aplicación de la rúbrica de la Tabla 1 en los dos cursos anteriores sobre la actividad Presentación del trabajo académico.

(c) ) EY-NC-ND 2018, Universitat Politècnica de València 


\section{Conclusiones}

La aplicación de las rúbricas es una herramienta potente para contextualizar la aplicación de las competencias transversales en las carreras técnicas.

En la presente contribución se ha descrito cómo pueden desarrollarse estrategias activas para que los alumnos se impliquen en actividades que involucren la comunicación efectiva, y como la puesta en marcha del proyecto institucional de la UPV de Competencias transversales, puede llevarse a la práctica en aspectos de evaluación a través de las rúbricas. Las rúbricas presentadas para la evaluación de la competencia a nivel de alumnos de últimos cursos de máster ha sido aplicada con resultados muy satisfactorios y ha permitido tener constancia de cómo los alumnos han sido capaces de llevarla a la práctica en una fase final ya de su educación superior.

\section{Agradecimientos}

La experiencia aquí descrita se ha llevado a cabo como parte de los trabajos desarrollados en el EICE DESMAHIA "Desarrollo de metodologías activas y estrategias de evaluación aplicadas al campo de la Ingeniería Hidráulica". Los autores agradecen a la UPV, y en particular al ICE, el apoyo prestado para llevar a cabo esta iniciativa.

\section{Referencias}

Fernandez March, A. (2010) La evaluación orientada al aprendizaje en un modelo de formación por competencias en la educación universitaria. REDU VOL 8(1).

Pérez-SÁnchez, Modesto; López Jiménez, Petra Amparo (2017a).El debate como instrumento complementario de aprendizaje en la competencia de responsabilidad ética, medioambiental y profesional. En Congreso Nacional de Innovación Educativa y Docencia en Red (IN-RED 2017). (683 - 695). Valencia, Spain: Editorial UPV.

Rodriguez, M. L. (2010). El debate como herramienta pedagógica. Plataforma de Metodología de la Investigación Científica y para la Guía de Tesis de Grado. Consultado en Internet en agosto de 2017 en: https://metodologiasdelainvestigacion.wordpress.com/2010/11/24/el-debate-comoherramienta-pedagogica/

UPV (2018). La comunicación efectiva como competencia transversal. Consutado en marzo de 2018 en: http://excelcon.blogs.upv.es/2015/02/24/la-comunicacion-efectiva-comocompetencia-transversal/

(cc) EY-NC-ND 2018, Universitat Politècnica de València 